\title{
HEALTHCARE SYSTEMS DESIGN: A SANDBOX OF CURRENT RESEARCH THEMES PRESENTED AT AN INTERNATIONAL MEETING
}

\author{
N. Ciccone ${ }^{1, \otimes}$, F. Patou ${ }^{1}$, A. Komashie ${ }^{3}$, G. Lame ${ }^{2}$, P. J. Clarkson ${ }^{3}$ and A. M. Maier ${ }^{1}$ \\ ${ }^{1}$ DTU-Technical University of Denmark, Denmark, ${ }^{2}$ University of Cambridge, United Kingdom, ${ }^{3}$ THIS. Institute, \\ United Kingdom \\ $\triangle$ nicic@dtu.dk
}

\begin{abstract}
Healthcare systems are under strain, this creates a challenge for designers to develop solutions for better health and care delivery. This paper presents a sandbox of illustrative design themes used to improve health systems based on state of the art research projects. These were collated from presentations at The Second International Meeting on Healthcare Systems Design Research, held at DTU-Technical University of Denmark. Attending groups were mapped based on their research keywords, target journals and methodologies in order to gain insight on the communities research landscape.
\end{abstract}

Keywords: healthcare design, complex systems, systems approach, healthcare, engineering design

\section{Introduction: Designing for future health and care}

Healthcare is a fundamental human need (Ghebreyesus, 2017). Goal 3 of the 17 United Nations Sustainable Development Goals (SDGs) calls to action for changes to ensure healthy lives and to promote well-being for all at all ages. Healthcare is a vital aspect of society and as such, progress towards better health and care has a symbiotic relationship with other aspects of sustainable development. Health is one of the greatest of today's societal challenges. As people age they become more susceptible to develop chronic conditions and as such, the resources required to deal with their medical needs increase (Denton and Spencer, 2010). The ageing of the population in the Western world, concurrently with greater need for access to healthcare drives higher costs. This weighs on economies, with higher GDP spending being needed to maintain quality and accessibility of care (WHO, 2018).

Designing better health and care is a continuous and iterative process, involving improvements in infrastructure, treatments, diagnostics and design frameworks (Pannunzio et al., 2019). Also, recent and rapid advances in technology have been transformational to healthcare improvement, greatly contributing to the increase in quality of life and lifespan of the last few decades (Cutler and McClellan, 2001). Technology has changed the experience of ill-health for the patient and their relatives and it has also had a radical impact on medical processes, driving fundamental change in healthcare professional practices (Hofmann, 2015). Yet, increasing technology-adoption costs, process inefficiencies, budget limitations, and scarcity of care personnel (Cutler et al., 2006; Spillman and Lubitz, 2000) all contribute to the faltering of healthcare systems performances. Today, only 50-60\% of care is being delivered in line with guidelines; around a third of medicine is wasteful considering its expenditure and the rate of adverse medical events remains approximately 1-in-10 patients (Braithwaite, 2018). 
These great challenges are increasingly acknowledged by clinicians and design researchers, engineers, innovation consultants, analysts and policymakers are also aware of this common responsibility to design for better health and care. The socio-technical complexity of healthcare means a more holistic approach is needed to explore, conceptualise and implement the most efficient healthcare interventions and procedures (Fidsa, 2009). For complex interventions specifically, those made of multiple components interacting in complex ways, integration and implementation often reveal great challenges, the overarching system in which the intervention is applied responding unpredictably in an attempt to adapt to the change (Shiell et al., 2008). As a system becomes more complex, nonlinearities emerge together with their unintended consequences (Kannampallil et al., 2011). This makes the creation of effective and predictable interventions difficult, requiring the collaboration of stakeholders from various backgrounds each bringing their unique capabilities and viewpoints.

Such different capabilities and viewpoints are reflected in a series of international meetings on healthcare systems design research and practice where contributors from engineering-, management/organisational- and clinical sciences have begun to come together. This paper reports on core results from the second meeting held in Denmark in 2019 (Feldman, 2019), following the first meeting held in the UK in December 2018 (THIS.Institue, 2018). Both meetings spanning two days were coorganised by the Cambridge Engineering Design Centre and The Healthcare Improvement Studies Institute from the University of Cambridge and the Engineering Systems Design group from the DTUTechnical University of Denmark. The international meetings are also intended as a launch platform for a wider interdisciplinary initiative towards a Healthcare Systems Design special interest group. The main aim and contribution of this paper is as an overview of 'sandbox cases': A rich portfolio of themes and capabilities created from data collected from participant researchers during the international healthcare meeting. By having health system design themes readily available to researchers and practitioners, we hope this can help tackle the complexity of designing for a healthy future. Together, the examples point to the opportunities of systems approaches to health and care (Clarkson, 2018; Ciccone et al., 2019 and Pannunzio et al., 2019) for value-effective outcomes (Patou and Maier, 2017). The remainder of the paper is organised as follows: Section 2 introduces the structure of the Second International Meeting of Healthcare Systems Design Research held at DTU-Technical University of Denmark. Section 3 reports on the 'sandbox cases' of research project themes. Section 4 reports on the research landscape mapping, and, looking forward, Section 5 provides a glimpse into next steps.

\section{Methods: Meeting workshop structure}

To contextualise the work reported in this paper, the First International Meeting on Healthcare Systems Design Research was conducted at the University of Cambridge in December 2018 (Komashie et al., 2019). The aim of the event was to identify the unique contribution systems design can make in achieving sustainable improvements in health and care delivery internationally, and to lay the foundations for a community of researchers and practitioners dedicated to healthcare systems design across disciplinary boundaries. Among the participants spanning multiple countries and disciplinary research communities, there was agreement with a strong demand from practitioners and policymakers for an overhaul through process-, organisational- and technological design in healthcare. However, the exchanges also pointed to the current lack of clarity and consensus on what healthcare systems design research might entail and how we might overcome current difficulties in defining and measuring impact of design interventions within complex healthcare systems. This suggested the value of exploring what it is that researchers participating in the two events are working on, and to begin to understand the joint capabilities and methodologies used in systems design research to navigate the complexity of healthcare. The aims of the Second International Meeting on Healthcare Systems Design Research were therefore threefold: 1 - to learn more about the research of attending participants; 2 - to map expertise and identify areas for collaboration and 3 - to plan future outputs for the community dedicated to healthcare systems design. The two-day event revolved around ignite talks and a set of discussion sessions. Preceding the meeting, participants completed a questionnaire with keywords pinpointing their main discipline through targeted journals and methodologies used. The meeting took place at the Skylab at the DTU-Technical University of Denmark in April 2019, with 34 participants from seven European countries, spanning roles including researcher, clinician, health innovation consultant, and 
policymaker. Each group that presented sent a copy of their ignite talk and questionnaire to the organisers who then collated them, creating themes on similar topic areas. This paper emphasises the research examples presented based on the ignite talks.

\section{Emerging research themes: A systems design sandbox}

Each participating research group was tasked to give a ca. 10-minute ignite talk. Prior to the meeting, participants were informed that the talk should include examples of their current systems design research projects, the main research questions addressed by research each group and some envisaged future directions. The purpose of the exercise was to paint a landscape of the research being conducted, laying the foundation for a systems design research 'sandbox'. Given that the ignite talks were meant to showcase examples of concrete research projects of those attending the meeting, the themes discussed below are selective and do not presume to give an exhaustive account of the potential of systems design in healthcare.

\subsection{Designing value-effective healthcare}

Value-based healthcare was first introduced as intending to incorporate the highest level of evidencebased data on patients' perceived value against the expenditures associated with a given health care intervention (Brown and Brown, 2005). Designing for value-effectiveness is therefore an attempt to maximise the perceived value across stakeholders given a clear account of required expenditures. The challenge of achieving value-effectiveness resides, in the variety of concept of value for the various stakeholders and the various system levels of our healthcare systems. A core system design task and question is what might be agreed as being value-effective and how may it be achieved? A proposal presented by the Engineering Systems Design group from DTU promotes value-effectiveness (Patou and Maier, 2017) by evaluating potential misalignments in the concept of value whenever undertaking the design of a product or service for healthcare. Several guiding principles are suggested to help in that endeavour, such as the consideration of design-for-change (e.g. design for evolvability) as a powerful tool or using a systemic approach founded on behavioural theory, technology and a contextual understanding of healthcare delivery systems (Ciccone et al., 2019). A design framework was proposed to set out the process and some of the indicators required to achieving this goal. Three entry angles were suggested for any healthcare improvement and newly designed initiative. This includes a broader and deeper understanding of the behaviour of those involved, the technological opportunities for addressing a particular issue and an analysis of the current healthcare system in place (Ciccone et al., 2019). This framework is currently being applied to technological advancements in stroke rehabilitation.

\subsection{Accounting for risk}

Risks to patients, staff, and organisations are prevalent in healthcare. The Engineering Design Centre, University of Cambridge, introduced a systems approach to Engineering Better Care (Clarkson, 2018) in the UK health service, heavily relying on an awareness of the importance of risk as a means to deliver safe, effective and affordable care. This framework considers people, systems, design and risk perspectives on a system, and how these complementary views could deliver benefits through systems thinking. Current projects implementing the latter approach include a collaboration with the Global Health Research Group on neuro-trauma to improve global neuro-trauma care. This is accomplished through a series of projects and activities focusing on various aspects of traumatic brain injury in low and middle-income countries, e.g. understanding trauma care in Myanmar and adapting the toolkit for use in global health (Nicolosi et al., 2018). Another example is the development of the Risk Identification Framework (RID Framework) to enhance improvement in risk identification (Simsekler et al., 2018). Risk at the patient level was considered, including how design can be used to improve patient wellbeing, through designing products, environments, information and systems (Kaya et al., 2019), with links made between the relationship of technology and risk, minimising patient and user harm.

\subsection{Gaining individual insight leading to systemic change}

A bottom up approach envisions intervention-design based at the level of the individual. Gaining insights into the needs of patients and healthcare workers can reshape the workings of the overall 
system. One of several strategies that can be implemented to engineer better care. At the Engineering Systems Design group at DTU, a research example is dementia monitoring and support, whereby smart wearables are capable of both supporting people with dementia and generating data about their behaviour could help rethink the way rehabilitation is planned and monitored (Thorpe et al., 2019). This project explored how behavioural sensing could be leveraged in a connected care system for active ageing to improve care through decentralisation of healthcare. Another example is collaborative care: an attempt to promote a re-balancing of patient/clinician information availability, decision-making power, and engagement and ownership between patient and care provider. Collaborative care is currently being investigated in the context of heart failure (Valentin-Hjorth et al., 2018). Likewise, the Copenhagen Centre for Health Technology (CACHET) with a portfolio of research projects aims to harness personal healthcare technologies to gain insight that can inform and influence systemic clinical workflows. A recent example is with the RADMIS - Treatment of Mental Health project which is divided into three goals: Research, design, and development of a smartphone-based monitoring and treatment platform for affective disorders. The platform is meant to provide clinical evidence on the number and duration of hospital re-admissions of patients with unipolar and bipolar disorder and to use smartphone-based cognitive behavioural therapy to improve symptoms of depression (FaurholtJepsen et al., 2017). Another example was contributed by Mälardalen University's Living Lab where users are encouraged to be involved in different stages of a design and/or innovation process. Co-design forms part of a tripartite relationship between the individual, university and companies. The purpose of which is to empower the end user while also fostering opportunities of collaborations between stakeholders in the private as well as in the public sectors.

\subsection{Using technological developments and data in healthcare}

Technological developments in healthcare are improving both patient quality of life and lifespan (Cutler and McClellan, 2001). Not only has technology changed the experience of ill-health for the patient and their relatives, but it has also had a radical impact on medical processes and driven change in healthcare professional practices (Hofmann, 2015). This permeability to affect multiple stakeholders and increases in richness of data obtained makes technology a potentially ideal starting platform to create systemic change. The importance of technology was, for example, embodied by one of the ignite talks introducing the role of a Clinical Engineer within the NHS, a professional group embedded within hospitals with a broad remit. The main objective of this position is to maximise value delivered by medical technology (Akinluyi et al., 2019). This links to the relevance of data science and the awareness of our community of the importance of harnessing data both in the context of research endeavours but also to support the design of products, interventions, and organisations for better health and care. The Open University encompassed this theme through their research in the various types of margins that are applied during building services design, the various stakeholders involved in a case study of a hospital boiler upgrade were highlighted. With safety margins being distinguished between those built into regulation, clinical, and contractual requirements, and those applied through the engineering design choices that allow for various contingencies and uncertainties. It was found that this resulted in over-design of core systems which often negated savings achieved by newer technology (Jones and Eckert, 2017). Sensors may also be used to inform a healthcare professional about the health of a patient but can also be used to support the design of products, interventions, and organisations for better healthcare. Thorpe et al. (2019) investigated how smart wearables (smartwatch, smartphone) might be adapted and used to support the functional, psychosocial and safety related needs of people with dementia while also deriving measures that describe users' behaviour (activity patterns, unusual/dangerous behaviour) which may have clinical significance.

\subsection{Using product and service development methodologies}

Development methodologies were introduced to help create value effective healthcare products- and services. Modelling of complex products and processes including functional, modular and platform- 
based developments were presented by the Systems Engineering Design group at Chalmers University of Technology (Borgue et al., 2019), together with the use of engineering strategies of modularisation and customisation and their applications in the precision delivery of medicines to improve efficiency (Siiskonen et al., 2019). Further examples using product- and service development methodologies included user-centred design for adapting smart wearables to the needs of seniors with mild cognitive impairment and the development and testing of pervasive assistive technology solutions created by combining off-the-shelf smart technology (Thorpe et al., 2016). Further examples include the use of visual design communication and prototyping (Eriksson and Fundin, 2018) for user involvement and co-creation of health and care solutions or for supporting the process of developing design methodologies for behavioural- and organisational change more widely (Daalhuizen et al., 2019). All of the above pay particular attention to various stakeholders, a vital aspect of system thinking. The importance of which is further brought to attention within the development of medical device design, e.g. how novice designers are using prototypes in practice, with a need of involvement of multiple stakeholders by DTU's section of Engineering Design and Product Development (Deininger et al., 2015).

\subsection{Modelling complex flows with participatory and numerical simulations}

Complexity is the "dynamic and constantly emerging set of processes and objects that not only interact with each other, but come to be defined by those interactions" (Cohn et al., 2013). Complex systems are characterised by undefined or changing boundaries, and many internal interactions adapting and co-evolving with other systems (Plsek and Greenhalgh, 2001). Understanding complexity 'offers ways to change the collective mind-set about healthcare systems, enabling improved performance through informed design that is otherwise stagnant' (Braithwaite, 2018). Participatory table-top simulation methods for co-design of healthcare work systems and user-driven healthcare innovations of hospital workflows, e.g. in an outpatient department as joint work between the Engineering Systems Design and Implementation and Performance Management research groups at DTU (Broberg and Edwards, 2012). The University of Leeds has used an understanding of the components of a complex system to improve forecasting and thereby pharmacy supply chain efficiencies (Phillips and Nikolopoulos, 2019). A further example of research in this area comes from researchers in healthcare logistics at KTH Royal Institute of Technology, pursuing research from the cellular and molecular level to that of the large-scale healthcare system. For example, gamification as one interactive modelling method was used. Simulation here mimics the behaviour of a real-world system, uses real people as decision makers that combined with (computerised) simulation models provides a rich source of insight for product design. A recent example is Simulation Game of Patient Transportation (Zhang and Sebastiaan, 2019). Simulation studies and computer modelling is also being conducted in Operational Research at Loughborough University as part of an Academic Health Science Centre, with computer models mapping patient flows, e.g. in an obesity service. Using these models different scenarios were tested to investigate the likely impact of alternative resource configurations on patient waiting times, impacting on the planning and organising of the obesity service (Tako et al., 2014). Finally, the benefit of simulation has been demonstrated by Loughborough University in the SIMTEGR8 project that developed and implemented simulation models, generating discussion amongst different stakeholders about the effectiveness of patient pathway analysis to improve care (Tako et al., 2019).

\section{Mapping and discussing the research landscape}

The 'sandbox' of examples from the ignite talks illustrates a snapshot into the rich portfolio of capabilities of healthcare systems design research. Having emphasised research examples that are currently being undertaken by the participants in the international meeting representing 15 institutions and 34 individuals from seven European countries, to understand the scope of research expertise and capabilities, results from a questionnaire that was distributed prior to the meeting were also discussed. Questions included how the participant categorise their overarching research discipline, what journals they target for publications, keywords that described their research and what methodologies/frameworks they use (Figure 1). 

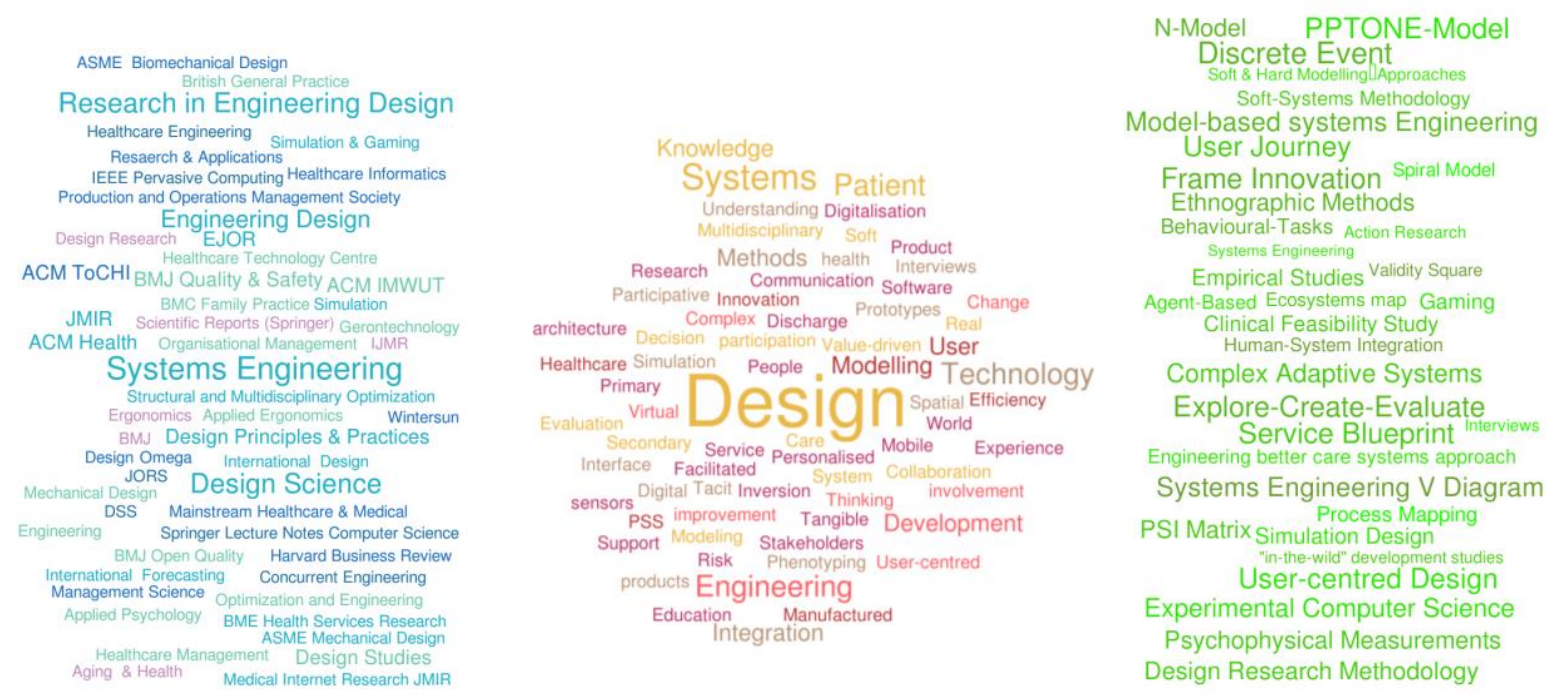

Figure 1. Questionnaire answers with disciplinary target journals (left), keywords describing research practice (centre) and research methodologies/frameworks used (right)

\subsection{Target journals, keywords from research practice and methodologies used}

Clusters of capabilities and interest can be inferred from responses in the questionnaire. Engineering design and technology hold a central place, with researchers publishing in journals, e.g. Gerontechnology, the Journal of Biomedical Design, IEEE Pervasive Computing, Systems Engineering, Design Science, Research in Engineering Design, Journal of Engineering Design, Journal of Design Research, Design Studies, Journal of Mechanical Design, and International Journal of Design. Keywords mentioned include 'technology integration', 'technology development', 'software architecture', and 'mobile sensors'. This emphasises the role of technology in supporting effective diagnosis and promoting better therapies and also contributions to resolving the systemic issues our healthcare systems suffer from.

Another cluster of capabilities and interest comes from management-/organisational sciences, with targeted journals such as the European Journal of Operational Research, Harvard Business Review, the Healthcare Engineering Journal, the Journal of Operational Research Society, the Journal of Organisational Management, the Journal of Healthcare Management, and Management Science. This is not surprising, as healthcare is essentially still delivered in large, complex organisations such as hospitals and clinics.

A third pole of capabilities and interest revolves around the clinical sciences, with target journals mentioned in the responses to the questionnaire, such as the British Journal of General Practice, the British Medical Journal or the Journal of Ageing and Health, or the Journal of Medical Internet Research (JMIR).

\subsection{System-level exercise}

Having mapped and discussed the main clusters of research disciplines and capabilities through visualising the questionnaire responses on disciplinary target journals, keywords describing research practice and methodologies/frameworks predominantly used in research, participants were then asked to position their work with sticky notes on a healthcare system model that emphasises four levels: patient, care team, organisation, and environment (Fanjang et al., 2005).

The model is of course a simplification and should be seen as a somewhat arbitrary gradient or continuum of levels rather than distinct layers. Therefore, most research initiatives marked on the sticky notes are placed across boundaries delineated in the patient-care team-organisationenvironment model presented in Figure 2. In positioning their work, multiple positions where chosen and it emerged in discussion that some researchers took a research project perspective, while others took a programme perspective in placing their sticky note. This shows that a focus 
unit of analysis and intervention might be on one level, while boundary conditions and impact pathways might stem from and connect with other system levels. Although such layered or continuum-based view of healthcare systems broken down into its constituents draws attention to the focus unit of analysis, a synergy of research work from various perspectives and their linkages is important to address the systems nature and complexity of healthcare.
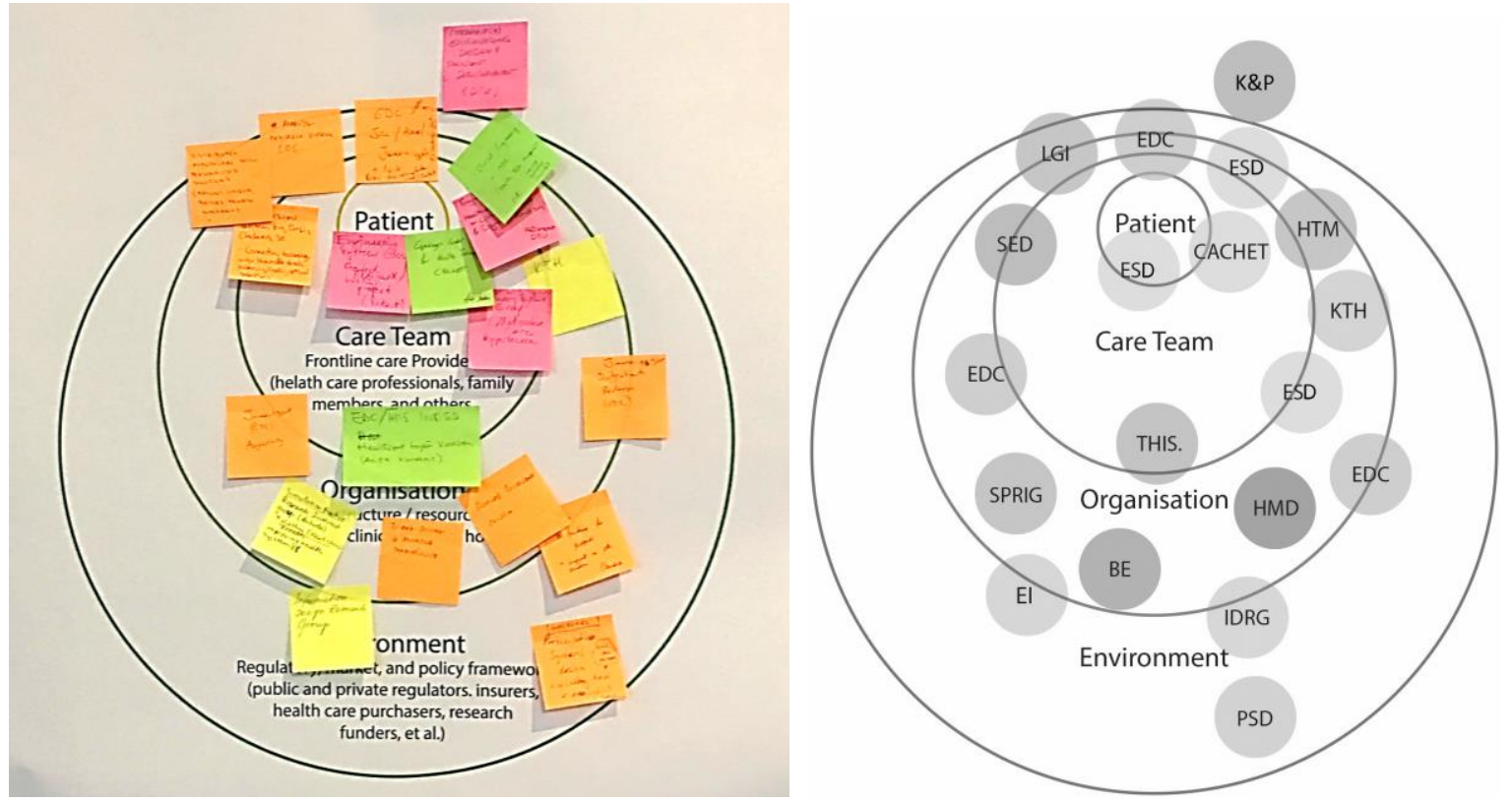

Abbreviations: ESD: Engineering Systems Design - DTU - Technical University of Denmark; CACHET: Copenhagen Center for Health Technology; KTH: Department of Biomedical Engineering and Health Systems KTH Royal Institute of Technology; SPRIG: Simulation Practice Research Interest Group - Loughborough University; IDRG: Information Design Research Group - Mälardalen University; THIS.: The Healthcare Improvement Studies Institute; EDC- Engineering Design Centre - University of Cambridge; SED: Systems, Engineering Design - Chalmers University of Technology; K\&P: Engineering Design and Product Development -DTU-Technical University of Denmark; PSD: Participative System Design - University of Leeds; EI:

Engineering and Innovation - The Open University, HTM: Healthtech Management - Guy's and St Thomas' NHS, BE: Business Excellence - University of Leeds, LGI: Leeds General Infirmary.

Figure 2. Four major levels of systems in healthcare delivery (Fanjiang et al., 2005) with research groups giving examples of their emphasis and target users of research through positioning sticky notes (left) and re-drawn for legibility (right); Not all research groups where present during the system-level exercise conducted on day two of the meeting

Overall, the discussion highlighted the necessity for clear system boundaries for any study in healthcare systems design research as well as the potentially resulting tension between clear and perhaps narrow delineation of scope for e.g. early clinical research and necessity of consideration of multiple levels for implementation of research results.

This brought the discussion to a particular focus on interaction with the clinical sciences. Our interest in the clinical sciences is an inevitable facet of pursuing design research for better health. After all, one may expect that the output of a design-for-health process (e.g. a medical device, a complex intervention, guidelines for better-outreach public health campaigns) would in many cases be evaluated by its direct clinical impact: by the benefits brought to patients. This, in turn, means the thorough pursuit of clinical research and the evaluation of success criteria (also known as clinical end-points) in the medical realm. At the boundary between the clinical, natural and social sciences, our community also shows interest in the behavioural and cognitive sciences as substrates for better understanding of the human aspects associated with the difficulty of promoting better healthcare in certain context, including for instance life-style diseases, poor treatment adherence, reluctance to embrace change, or cognitive biases in the medical community. 


\section{Conclusions and outlook}

The Second International Meeting on Healthcare Systems Design Research at DTU-Technical University of Denmark brought together 15 different institutions across Europe, represented by 34 people. Participants shared their research expertise and interests. A rich portfolio of research' sandbox' cases and understanding of contributing research disciplines through target journals, a selection of keywords and research frameworks used was highlighted in this paper. Moreover, mapping of research project- and programme focus on multiple system levels including patient, team, organisation, and environment was undertaken. The rich portfolio, breadth of expertise and systems' mind-set encourages in believing that we meet the essential requirements to address and navigate the complexity of healthcare. A sandbox of illustrative themes based on the participants' research projects that could help achieve this was stated: designing for value-effectiveness, accounting for risk, gaining individual insight leading to systemic change, using technological developments and data in healthcare, using product and service development methodologies and modelling complex flows with participatory and numerical simulations. In going forward, a third international meeting is planned, bringing results from the two already held meetings together and focusing on detailing the research project portfolio and use cases with the view towards an edited volume on health systems design research and practice. Attention will be also paid to other groups in this area and their current contributions, including work within more private based health systems. This will lay the foundations necessary for operationalising system thinking to navigate and combat the ever-growing strains healthcare is facing globally.

\section{References}

Akinluyi, E.A., Ison, K. and Clarkson, P.J. (2019), "Mapping outcomes in quality improvement and system design activities: the outcome identification loop and system impact model", BMJ Open Quality, Vol. 3 No. 8, p. 439.

Borgue, O., Panarotto, M. and Isaksson, O. (2019), "Modular product design for additive manufacturing of satellite components: maximising product value using genetic algorithms", Concurrent Engineering. https://doi.org/10.1177/1063293X19883421

Braithwaite, J. (2018), "Changing how we think about healthcare improvement", British Medical Journal, Vol. 361. https://doi.org/10.1136/bmj.k2014

Broberg, O. and Edwards, K. (2012), "User-driven innovation of an outpatient department", Work, Vol. 41, pp. 101-106. https://doi.org/10.3233/WOR-2012-0142-101

Brown, M.M. and Brown, G.C. (2005), "Evidence-based to value-based medicine”, Amer Medical Assn.

Ciccone, N.W., Patou, F. and Maier, A.M. (2019), "Designing for better healthcare: A systemic approach utilising behavioural theory, technology and an understanding of healthcare delivery systems", Proceedings of the Design Society: International Conference on Engineering Design, Vol. 1 No. 1, pp. 937-946. https://doi.org/10.1017/dsi.2019.98

Clancy, T.R. and Delaney, C.W. (2005), “Complex Nursing Systems”, Journal of Nursing Management, Vol. 13 No. 3, pp. 192-201. https://doi.org/10.1111/j.1365-2834.2004.00518.x

Clarkson, P.J. (2018), "What has engineering design to say about healthcare improvement?", Design Science, Vol. 4. https://doi.org/10.1017/dsj.2018.13

Cohn, S. et al. (2013), "Entangled complexity: why complex interventions are just not complicated enough", $J$ Health Serv Res Policy, Vol. 18 No. 1, pp. 40-43. https://doi.org/10.1258/jhsrp.2012.012036

Cutler, D.M. and McClellan, M. (2001), "Is technological change in medicine worth it?", Health Affairs, Vol. 20 No. 5, pp. 11-29. https://doi.org/10.1377/hlthaff.20.5.11

Cutler, D.M., Rosen, A.B. and Vijan, S. (2006), "The Value of Medical Spending in the United States", New England Journal of Medicine, Vol. 355 No. 9, pp. 920-927. https://doi.org/10.1056/NEJMsa054744

Deininger, M. et al. (2015), "Novice designers' use of prototypes in engineering", Design Studies. https://doi.org/10.1016/j.destud.2017.04.002

Denton, F.T. and Spencer, B.G. (2010), "Chronic health conditions: changing prevalence in an aging population and some implications for the delivery of health care services", Canadian Journal on Aging/La Revue canadienne du vieillissement, Vol. 29 No. 1, pp. 11-21. https://doi.org/10.1017/S071498080999039

Daalhuizen, J. et al. (2019), "An architecture of design doing: A framework for capturing the ever-evolving practice of design to drive organizational learning", International Journal of Design, pp. 37-52.

Eriksson, Y. and Fundin, A. (2018), "Visual management for a dynamic strategic change", Journal of Organizational Change Management, pp. 712-727. https://doi.org/10.1108/JOCM-05-2016-0103 
Fanjiang, G. et al. (2005), Building a better delivery system: a new engineering/health care partnership, National Academies Press. https://doi.org/10.1108/0-309-09643-X

Faurholt-Jepsen, M. et al. (2017), "Reducing the rate and duration of Re-ADMISsions among patients with unipolar disorder and bipolar disorder using smartphone-based monitoring and treatment -- the RADMIS trials: study protocol for two randomized controlled trials", Trials, Vol. 277. https://doi.org/10.1186/s13063017-2015-3

Feldman, A. (2019, May), How to design future healthcare using a systems approach, Retrieved from http://www.innovation.man.dtu.dk/english/research/engineering-systems/news/Nyhed?id=\{70643834-3FE54D9A-B944-DB9F139C450D\}

Feldman, A. et al. (2019), "Listening Effort and Cognitive Decline: An Exploratory Study Using Pupillometry", International Conference on Cognitive hearing science for communication.

Fidsa, G.Z. (2009), "Designing the future of healthcare”, Stud Health Technol Inform, Vol. 149, pp. 49-57.

Ghebreyesus, T. A. (2017), Health is a fundamental human right. World Health Organization.

Greenhalgh, T. and Papoutsi, C. (2018), "Studying complexity in health services research: desperately seeking an overdue paradigm shift", BMC Medicine. https://doi.org/10.1186/s12916-018-1089-4

Hofmann, B. (2015), "Too much technology”, British Medical Journal, Vol. 350. https://doi.org/10.1136/bmj.h705

Hood, L. and Friend, S.H. (2011), "Predictive, personalized, preventive, participatory (P4) cancer medicine", Nature reviews, Vol. 184. https://doi.org/10.1038/nrclinonc.2010.227

Innes, A.D., Campion, P.D. and Griffiths, F.E. (2005), "Complex consultations and the "edge of chaos"”, BrJ General Practice, Vol. 55 No. 510, pp. 47-52.

Jones, D. and Eckert, C. (2017), "Overdesign in building services: the hidden energy use", 21st International Conference on Engineering Design.

Kannampallil, T.G. et al. (2011), "Considering complexity in healthcare systems", Journal of biomedical informatics, pp. 943-947. https://doi.org/10.1016/j.jbi.2011.06.006

Kaya, G.K., Ward, J. and Clarkson, J. (2019), "A Review of Risk Matrices Used in Acute Hospitals in England", Risk Analysis, pp. 1060-1070. https://doi.org/10.1111/risa.13221

Komashie, A. et al. (2019), "Exploring Healthcare Systems Design Research and Practice: Outcomes of an International Meeting", Proceedings of the Design Society: International Conference on Engineering Design, Vol. 1 No. 1, pp. 947-956. https://doi.org/10.1017/dsi.2019.100

Ludden, G.D. and Hekkert, P. (2014), "Design for healthy behavior: design interventions and stages of change", 9th International Conference on Design \& Emotion, pp. 482-488.

Nicolosi, F. et al. (2018), "Neurosurgical digital teaching in low-middle income countries: beyond the frontiers of traditional education”, Neurosurgical focus, No. 4, p. 45. https://doi.org/10.3171/2018.7.FOCUS18288

Niedderer, K. et al. (2014), "Creating sustainable innovation through design for behaviour change", Summary report.

Pannunzio, V., Kleinsmann, M. and Snelders, D. (2019), "Three approaches to design engineering in the health domain: a systemic perspective", Proceedings of the Design Society: International Conference on Engineering Design, pp. 1005-1014. https://doi.org/10.1017/dsi.2019.106

Patou, F. and Maier, A. (2017), "Engineering value-effective healthcare solutions: A systems design perspective", Proceedings of the 21st International Conference on Engineering Design (ICED 17), pp. 31-40.

Phillips, C.J. and Nikolopoulos, K. (2019), "Forecast quality improvement with Action Research: A success story at PharmaCo", International Journal of Forecasting. https://doi.org/10.1016/j.ijforecast.2018.02.005

Plsek, P.E. and Greenhalgh, T. (2001), "Complexity science - the challenge of complexity in health care", British Medical Journal, Vol. 323, pp. 625-628. https://doi.org/10.1136/bmj.323.7313.625

Shen, L. (2010), "Mitigating Psychological Reactance: The Role of Message-Induced Empathy in Persuasion", Human Communication Research, pp. 397-422. https://doi.org/10.1111/j.1468-2958.2010.01381.x

Shiell, A., Hawe, P. and Gold, L. (2008), "Complex interventions or complex systems? Implications for health economic evaluation", British Medical Journal, Vol. 336, pp. 1281-1283. https://doi.org/10.1136/ bmj.39569.510521.AD

Siiskonen, M. et al. (2019), "Decision Support for Re-designed Medicinal Products-Assessing consequences of a customizable product design on the value chain from a sustainability perspective", Proceedings of the Design Society: International Conference on Engineering Design, pp. 867-876. https://doi.org/10.1017/ dsi.2019.91

Simsekler, M.E., Ward, J.R. and Clarkson, P.J. (2018), "Design for patient safety: a systems-based risk identification framework", Ergonomics, Vol. 8 No. 61, pp. 1046-1064. https://doi.org/10.1080/00140139. 2018.1437224

Spillman, B.C. and Lubitz, J. (2000), "The Effect of Longevity on Spending for Acute and Long-Term Care", New England Journal of Medicine, Vol. 342 No. 19, pp. 1409-1415. https://doi.org/10.1056/ NEJM200005113421906 
Tako, A. et al. (2014), "Improving patient waiting times: a simulation study of an obesity care service", BMJ Qual Saf, pp. 373-381. https://doi.org/10.1016/j.dss.2011.11.015

Tako, A. et al. (2019), "Evaluating community-based integrated health and social care services: The Simtegr8 approach", Simulation for Risk Management, pp. 1-12.

THIS.Institue. (2018, December), blog.thisinstitute, Retrieved from THISInstitute Cambridge: https://blog. thisinstitute.cam.ac.uk/putting-pieces-together-healthcare-systems-design

Thorpe, J.R., Forchhammer, B.H. and Maier, A.M. (2019), "Development of a Sensor-Based Behavioral Monitoring Solution to Support Dementia Care", JMIR mHealth and uHealth, No. 7, p. 120. https://doi.org/10.2196/12013

Thorpe, J.R. et al. (2016), "Pervasive assistive technology for people with dementia: a UCD case", Healthcare technology letters, pp. 297-302. https://doi.org/10.1049/ht1.2016.0057

Valentin-Hjorth, J.F. et al. (2018), "Design for health: towards collaborative care", DESIGN2018-15th International Design Conference.

WHO. (2018), European Health Information Gateway: Total health expenditure as \% of GDP. Retrieved from https://gateway.euro.who.int/en/indicators/hfa_566-6711-total-health-expenditure-asofp/visualizations/\#id=19661\&tab=notes

Zhang, C. and Sebastiaan, M. (2019), “A Simulation Game of Patient Transportation”, In Ryoju Hamada;Songsri Soranastaporn; Hidehiko Kanegae; Pongchai Dumrongrojwatthana; Settachai Chaisanit; Paola Rizzi; Vinod Dumblekar. Neo-Simulation and Gaming Toward Active Learning, pp. 53-66. 